\title{
Packing materials for enantioselective preparative chromatography
}

\author{
G.B. Cox \\ Chiral Technologies Europe, Parc d'Innovation, boulevard Gonthier d'Andernach, 67404 Illkirch, France
}

This paper considers the application of chiral stationary phases to preparative liquid chromatography. The various types of CSPs are briefly reviewed in terms of their loading capacity, structure and general areas of application. Enantioselective preparative separations display certain aspects not necessarily encountered in more conventional achiral applications. These specifically involve the solubility of the target molecules in the mobile phases conventionally used in enantioselective chromatography. Strategies for the development of separations using mobile phases more suited to the requirements of preparative chromatography are outlined, with special reference to the use of carbohydratebased CSPs. Since the selectivity is the most important parameter in determining the usefulness of a particular set of separation conditions for preparative application, attention is also given to the optimisation of mobile phase selectivity by variation of the nature of the solvent and also of the acidic or basic additives which may be used in the case of the separation of ionogenic solutes under "normal phase" conditions. In the scale-up of preparative separations to production scale, which is becoming of increasing importance for chiral pharmaceutical products and intermediates, an often misunderstood parameter is that of the influence of particle diameter, especially in the case of SMB chromatography. The influence of particle size on the economics of large scale purifications is discussed and it is shown that, in contrast to the case of achiral separations where there is an economically optimum particle size, the particle diameter used for preparative enantioselective separations, even SMB, should be the smallest possible consistent with good column performance.

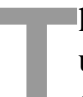

he past few years have seen a dramatic growth in the use of preparative separations of enantiomers. This has occurred due in part to the growing realisation that although one enantiomer of a pharmacologically active species may be beneficial, the other enantiomer may have zero or a negative and perhaps harmful effect. This has also been reflected in changes in legislation and practices in the pharmaceutical industry where ever more emphasis is being placed upon the preparation of enantiomerically pure drug substances, either for new products or in order to make "racemic switch" products, replacing an existing racemic product with the pure active enantiomer.

Although in the first instance the emphasis has been upon the analytical separation of enantiomers in order to follow the more traditional processes of crystallisation and asymmetric synthesis to prepare the enantiomerically pure products, more and more preparative separations are being developed for optical resolution of racemic mixtures. This is for a number of reasons. One, of major importance, is that of time. A chromatographic purification can be developed very rapidly and can be in operation quickly. In the early stages of development, this is important so that initial evaluation of activity and toxicity can be made before time is invested in the often lengthy development of an asymmetric synthesis. In some cases, this factor of rapid access to the enantiomerically pure products can be sufficiently important to justify the continuation of the use of chromatography further into the development process. In other cases, especially since the introduction of techniques such as Simulated Moving Bed Chromatography, it has been found that the chromatographic process is less expensive than the use of the traditional techniques and production scale chromatography is used for the manufacture of the enantiomerically pure drug product.

The optimisation of preparative separations is critically dependent upon the nature of the packing material used for the purification, especially if the separation is likely to be used in the manufacturing process (it can be argued that the 
speed of the chromatographic resolution outweighs all other considerations at the early stages of development). Different packings have different chiral selectors and different modes of operation. Some of these are more easily adapted to the preparative process than others, in that they are more robust, have higher capacities and are more selective than their counterparts. There are, however, always compromises to be reached in the selection of chiral stationary phases (CSPs) for preparative and production scale operation. This paper considers some of these aspects and describes some typical applications for a range of useful CSPs as well as considering some of the economic aspects of their application to production scale LC.

\section{Requirements for CSPs for preparative and production scale chromatography}

Preparative chromatography places demands upon packing materials beyond those which apply to packings devoted to analytical chromatography. Because preparative columns are of larger diameter than the analytical columns, the particles are subjected to higher stress [1] and should therefore be robust. Allied to this is the requirement that the packings should allow high flow rates at reasonable operating pressures. The solution to this is to use spherical packing materials with carefully chosen particle size (see later) and of narrow particle size distribution. These physical properties of the packing materials are not, however, the object of the present discussion since there are other properties of the CSP that influence the usefulness of the material in preparative operation.

A major concern in preparative chromatography is that of selectivity. It is well known that selectivity optimisation is the most rapid way to maximise the sample loading on a preparative column. If the maximum loading is to be achieved, it has been shown that this is related to selectivity by a complex relation [2] but which can be simplified to show that the loading factor (and the optimum production rate) for values of $\alpha$ above 1.03 follows to a first approximation the relation:

$$
L_{\mathrm{f}}=K\left(\frac{\alpha-1}{\alpha}\right)^{2}
$$

Figure 1 shows the results of this relation. The relative load is plotted against the selectivity. Other data indicates that a similar relation holds between the production rate and selectivity. It is clear that a small change in selectivity results in a large change in the possible load.

Selectivity, however, is only part of the picture. It is of little use having a very high selectivity if the column is rapidly overloaded, thus losing the separation as soon as the load is increased beyond the analytical scale. The estimation of the loadability of a column is not simple, since it depends very much upon the competitive isotherms of adsorption of the solutes on the CSP. Much work for the simple case - of Langmuir adsorption isotherms - has been done and this situation is well documented in the literature of preparative chromatography [3]. In this case, the loadability of the column can be defined in terms of the saturation capacity of the CSP. The saturation capacity is defined as the quantity

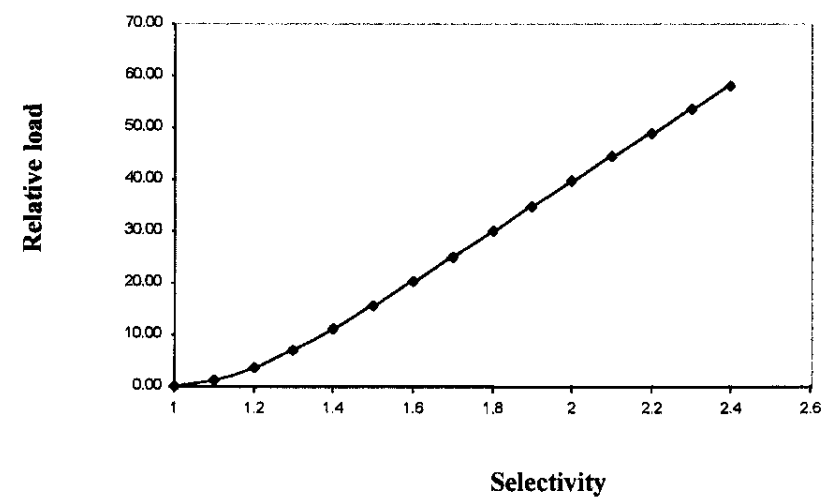

Figure 1. Plot of column loading versus selectivity. Capacity factor for the 1st component taken to be 3 .

of solute adsorbed on the surface of the packing at infinite solute concentration and corresponds to a monomolecular layer of the solute on the surface of the CSP [4]. Where Langmuir behaviour is realised, this parameter is of great value in assessing the capacity of a phase. The difficulty is that for the majority of CSPs one rarely finds the classical isotherm behaviour. There has been little work on the determination of competitive adsorption isotherms in enantioselective separations. One paper in this area considered the preparative separation of the enantiomers of N-benzoylphenylalanine on a protein-based CSP [5]. This separation was shown to be well represented by a bi-Langmuir isotherm. Such behaviour is typical where separate sites are responsible for overall retention and for the chiral selection. The distinction is rarely experienced on the majority of CSP types since there are not clear sub-sets of adsorption sites and the retention and chiral selection occurs at the same part of the phase. In such cases, the more retained enantiomer is considered to interact more strongly with the CSP and will probably take up more of the surface area than the less retained enantiomer. Thus, it is possible that (provided the individual isotherms follow the Langmuir model) the saturation capacities of the two enantiomers will differ. This situation can be described by combination of the individual isotherms by use of the Ideal Adsorbed Solution Theory [6]. In some circumstances this is an adequate representation of reality. As has been pointed out [7], the results obtained under such circumstances can be quite different from those from the competitive Langmuir isotherm. Unfortunately, when the second component has the lower saturation capacity, the displacement effects found so often in achiral chromatography are minimised and one frequently finds the socalled tag-along effects predominating. In evaluating the overall usefulness of types of CSP, however, it is possible to use the saturation capacities estimated from one or two separations to arrive at an approximate idea of the order of magnitude of the loading capacity of the CSP. It is equally important that the form of the isotherm for the CSP is reasonable: a bi-Langmuir isotherm, for example, usually gives rise to excessive peak tailing which can result either in poor purity or a very low sample load.

\section{Types of CSP}

CSP types range from very specific to the general. The capacity of these phases also varies in accord with the 
concentration of adsorption sites on the CSP. Where these have been measured, or data exists which can be used to calculate an approximate value, these are reported in table I. Although a ranking on such data is at best approximate, it nevertheless gives some idea of the relative merits of the CSPs for preparative purposes.

\section{Molecular imprinting}

CSPs made by molecular imprinting are very specific phases and can display large selectivity [8]. They are made essentially by assembling the CSP around template molecules (usually the species desired to be isolated), usually by a polymerisation of a monomer which has functional groups which act as sites of interaction with the functional groups in the target molecule. After this, the template molecule is removed. If the phase has been well designed, there are then specifically shaped sites in the CSP that can accept only the desired enantiomer. The disadvantage of such phases is that because the sites need to be relatively inflexible and cannot overlap, their concentration on the surface of the CSP is strictly limited; this currently restricts the loadability of the phases and their use in preparative chromatography is very limited.

\section{Protein phases}

Proteins have stereoselective interaction sites which are used in their natural interactions in physiological systems. This has been exploited for some proteins which can be immobilised on silica surfaces without significantly changing their structure. Bovine Serum Albumin, for example, has been used as a CSP component. The problem with protein phases is that they are essentially restricted to reversed phase separations due to the denaturation of the protein in non-aqueous systems. A further disadvantage for preparative chromatography lies in the fact that each large protein molecule has on average only a single interaction site for the chiral selection. This seriously limits the number of possible sorption sites in the CSP and this again limits the loadability of the phase.

\section{Cyclodextrin phases}

CSPs made from bonding cyclodextrin moieties to the surface of silica have been available for some time. These rely upon the insertion of the target molecule into an enantioselective cavity in the cyclodextrin ring. These CSPs have not been extensively used for preparative work, in part again because the size of the receptor molecule, whilst being considerably smaller than proteins, nonetheless necessitates the use of a certain space on the silica support surface, which limits the loadability.

\section{Poly(meth)acrylamides}

These CSPs, which are based upon a polyacrylamide which is synthesised from a chiral monomer, were first introduced by Blaschke and collaborators [9]. A variety of groups may be used to introduce chirality in the monomer, usually through the use of a chiral amine for the amide synthesis. These phases have reasonably high capacities, since the chiral selector is repeated at each monomer molecule. Provided sufficient pore diameter is available for access of the solute molecules, given that the surface of the support is covered
Table I. Saturation Capacities for CSPs.

\begin{tabular}{lc}
\hline Type of Phase & Saturation Capacity $(\mathrm{mg} / \mathrm{g})$ \\
\hline$\alpha$-Cyclodextrin [17] & 0.05 \\
BSA $^{5}$ & 1.575 \\
Whelk-O $^{\circledR}$ Chiralpak & \\
CD $^{\circledR}$ & $40-80$ \\
\hline
\end{tabular}

with a layer of cross-linked polymer, then these phases operate with reasonably good performance.

\section{$\pi$-acid and $\pi$-base - "Pirkle"- phases}

Phases specifically designed to allow the three-point interactions proposed to be required for enantioselectivity were synthesised by the group of Professor Pirkle from the beginning of the 1980s [10]. They were intended to represent a rational approach to the development of CSPs to allow the design of specific phases for any given task. Although this goal of predictability has eluded the researchers, this has not prevented the development of very many materials, some of which show a high selectivity. The Pirkle CSPs include a variety of functional groups which are selected to give a specific interaction - hydrogen bonding, charge-transfer, steric, etc.- and, as such, tend to be rather application-specific in selectivity, although one or two such phases, such as the Whelk-O material are claimed to have a wider applicability than most. The phases are usually bonded chemically to the support and are thus stable to any of the solvents one may wish to use to optimise the selectivity or the sample solubility. In addition, the Pirkle phases generally exhibit a reasonable sample capacity and efficiency, since they are usually prepared from high surface area silica and the bonded phase molecules are relatively small.

\section{Carbohydrate derived phases}

Probably the most used CSP in preparative separations has been cellulose triacetate [11]. This simple ester of cellulose in its microcrystalline form gives good separations of many enantiomeric species. Its major disadvantage in preparative chromatography is that given its physical form, the chromatographic efficiency of the material is disappointing and long retention times and high selectivity are required for the separation in order to counter the width of the chromatographic peaks. Other esters of cellulose, most notably the benzoate and p-methylbenzoate, have been used. These materials are available as beads of polymer [12] which have a much improved mass transfer over the cellulose triacetate. CSPs derived from these and other cellulose derivatives coated on silica have been developed and reported by Okomoto [13]. Such packings are very useful in preparative LC due to their efficiency and versatility. In order to allow a high loading of the polymer and to allow access to the internal pores in the particle, the pore diameter of the silica support is large (approximately 1200 Angströms). Despite the low surface area that this entails, the polymer coating is present to around $20 \%$, allowing a high sample loading, commensurate with other enantioselective phases used in preparative LC. The carbohydrate phases derive their enantioselectivity from their tertiary structure. The substituent 
groups, introduced by esterification of the three remaining hydroxyl groups on each sub-unit or by reaction of these groups with an isocyanate to make carbamate linkages, are present to provide some interaction with the solute molecules to generate retention and some orientation with respect to the chiral environment. Thus, these phases are amongst the more general of the CSPs used in chromatography at present.

\section{Optimisation of enantioselective preparative chromatography}

In order to achieve acceptable production rates in preparative chromatography, a number of parameters have to be optimised. The major of these, selectivity, has already been mentioned. Other parameters are also important. Probably the major challenge in the development of preparative enantioselective separations is that of achieving an appropriate solubility. The majority of separations of enantiomers are performed under conditions of low or even marginal solubility; the use of binary mixtures of hexane and an alcohol or other polar modifier is normal. The vast majority of pharmaceutically interesting molecules, however, are sparingly soluble in such solvents. This means that in order to introduce a reasonable quantity of solute, one is constrained to use very large injection volumes. This means that, unless other steps can be taken to improve the solubility, the selectivity is used in compensating the volume overload rather than in allowing a high mass load of solute. In batch chromatography, this can to some extent be ameliorated by the use of an injection solvent stronger than the mobile phase. Provided the volume is not excessive and that the solute does not immediately precipitate on entering a column filled with the weaker mobile phase, one can often increase the load. This cannot be done in Simulated Moving Bed systems since the feed solvent has to be the same as the mobile phase in order to keep the system in equilibrium as the mobile phase is recycled. This means that the solvents used for the process have to be carefully selected to ensure a maximum solubility whilst continuing to give a good selectivity and reasonable retention times.

\section{Selectivity}

Selectivity optimisation is carried out by changing the nature of the CSP as well as that of the solvent. The usual approach is first to select the CSP that shows at least some separation. Because there are only a few rules for selection of CSP, the usual method is to run a screening test on a range of CSPs to identify candidates. Some typical results are shown in table II for such a screening using thalidomide and four CSPs, two based on cellulose and two on amylose [14]. Once a useful CSP is identified, the next step depends greatly upon the type of CSP used and the scale of operation. At the laboratory scale, there is little incentive to optimise further the CSP unless there is no separation of the desired enantiomers. For industrial scale purifications, there may be good reason to spend some time in the optimisation of the CSP. Again, there are few rules to guide such optimisation and it is often performed by trying related CSPs with small changes in the chiral selector or in the surrounding groups. Where the separation is improved, it is
Table II. Retention data for Thalidomide on Cellulose and Amylose CSPs.

\begin{tabular}{lccc}
\hline CSP & $k_{1}^{\prime}$ & $k_{2}^{\prime}$ & $\alpha$ \\
\hline Chiralpak AD & 3 & 9 & 3 \\
Chiralcel OK & 2.2 & 3.3 & 1.5 \\
Chiralcel OJ & 0.6 & 0.9 & 1.4 \\
Chiralcel OB & 0.6 & 0.8 & 1.3 \\
\hline
\end{tabular}

then sometimes possible to design a new phase that is better suited for the purpose. It is also possible to use a CSP which is based upon the enantiomer to be isolated to select a chiral selector. The desired compound is immobilised on the silica support and the resulting CSP is used to perform enantioselective separations on candidate selectors for the CSP to be used in the purification. If a very high selectivity for one of these separations is found, it is expected that the reverse situation, where the solute from this latter experiment is used to form the CSP, will also result in a high selectivity.

In common with other modes of liquid chromatography, enantioselective separations are profoundly influenced by solvent selectivity effects. In the main, these effects are easier to optimise than is the nature of the CSP, since here no syntheses are required and there are therefore no potential problems of phase reproducibility or prolonged development time.

In the past, the majority of enantioselective separations have been carried out using binary hydrocarbon - alcohol mixtures. As noted earlier, most compounds have relatively low solubility in such mobile phases and for preparative purposes this solvent mixture is not especially useful. In fact, the only restrictions on solvent selection are that the CSP should be stable to the mobile phase and that the solvent should result in some selectivity for the enantioselective separation. Recent work has demonstrated that carbohydrate based CSPs have a much wider solvent stability than has previously been realised. Table III shows some possible solvent combinations that have been used with a variety of the Daicel phases that are available for preparative purposes. The differences in selectivity possible by changing the mobile phase composition are shown in table IV, in this case illustrating the separation of the enantiomers of WielandMiescher Ketone. For this separation, it is clear that ethanol gives the highest selectivity. In practice, the high viscosity of ethanol and the longer retention times observed may lead to use of a compromise mobile phase which has a lower viscosity and thus allows a higher rate of production for a given operating pressure even though it may give a slightly lower selectivity. The choice of mobile phase in this case will be determined by the scale and the economics of the operation.

Once the range of solvents is exhausted, there remain some other resources to improve the selectivity of the separation. Even in non-aqueous solvents, ionic effects can be important in changing selectivity. For many basic compounds, the chromatography and often the selectivity can be improved by the addition of a low concentration of an amine ( $0.1 \%$ diethylamine is frequently used). This does more than merely remove the silanol group interactions, since for some 
Table III. Solvents used for enantioseparations using carbohydrate-based phases.

\begin{tabular}{|c|c|c|c|c|c|c|}
\hline \multirow[b]{2}{*}{$C S P$} & \multirow[b]{2}{*}{ Acetone } & \multirow[b]{2}{*}{ Chloroform } & \multirow[b]{2}{*}{ Ethyl acetate } & \multicolumn{3}{|c|}{ Maximum \% (in Heptane) } \\
\hline & & & & Ethanol & Methanol* $^{*}$ & Acetonitrile* \\
\hline Chiralce $^{\circledR}$ OD & 20 & 5 & 20 & 100 & 100 & 100 \\
\hline Chiralpak $^{\circledR} \mathrm{AD}$ & 5 & 20 & 5 & 100 & 100 & 100 \\
\hline Chiralcel $^{\circledR}$ OJ & 20 & 10 & 5 & 100 & 100 & 40 \\
\hline Chiralpak $^{\circledR}$ AS & $\mathrm{ND}^{+}$ & $\mathrm{ND}^{+}$ & $\mathrm{ND}^{+}$ & 100 & 100 & 100 \\
\hline
\end{tabular}

${ }^{*}$ Note - not miscible with heptane.

+ ND - Data not available.

Table IV. Effect of mobile phase composition on the separation of the enantiomers of Wieland-Mieschler Ketone.

\begin{tabular}{lccc}
\hline Solvent & $k_{1}^{\prime}$ & $k_{2}^{\prime}$ & $\alpha$ \\
\hline Ethanol & 1.3 & 3.3 & 2.5 \\
Acetonitrile & 0.7 & 1.3 & 1.8 \\
Acetonitrile : 2-Propanol 1:1 & 0.35 & 0.7 & 2.0 \\
\hline
\end{tabular}

separations an increase in retention and selectivity can be observed on the addition of the amine, although one would normally expect these to diminish. An alternative for preparative separations can be to use an acidic additive instead of the amine. Figure 2 shows the separation of the enantiomers of pindolol [15] under the two sets of conditions. Even though the addition of TFA clearly results in very distorted isotherms, the situation from the point of view of the preparative separation is much improved, with the throughput increasing from 322 to $860 \mathrm{~g}$ of racemate per kilo of CSP per day. In some cases, both acid and base can be used to develop the selectivity. Figure 3 illustrates the effect of the addition of a small concentration of trifluoroacetic acid to a basic mobile phase in the chromatography of albuterol [16].

Unfortunately, the optimisation of the selectivity may not be the only priority in the selection of the solvent. In the separation of thalidomide, for example, the solubility in methanol is sufficiently poor $(1 \mathrm{~g} / \mathrm{L})$ that a compromise has to be reached. Thalidomide is much more soluble in acetonitrile $(5 \mathrm{~g} / \mathrm{L})$ and although the separation is rather poor in acetonitrile-rich solvents there is an optimum situation in which one uses a mixture of methanol - acetonitrile as mobile phase that allows the introduction of the solute in a solvent containing enough acetonitrile to allow a more reasonable solubility. The improvement in solubility, and thus the reduction in injection volume, more than compensates for the lower selectivity in the methanol acetonitrile mixture with the result that the production rate for the purification is improved.

\section{Migration to larger scale}

The passage from laboratory to production scale separations is simple for separations already completely optimised: one simply increases the column diameter until the desired production rate is reached. Unfortunately, there is much work a.

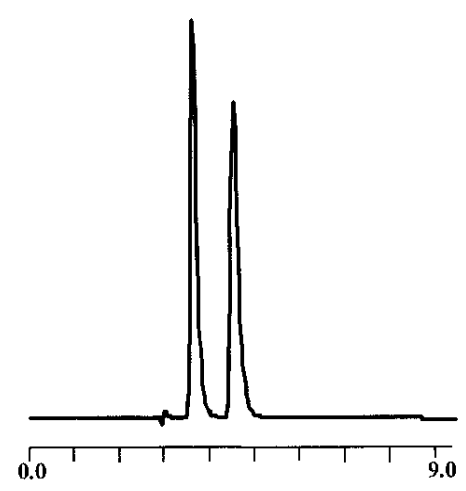

b.
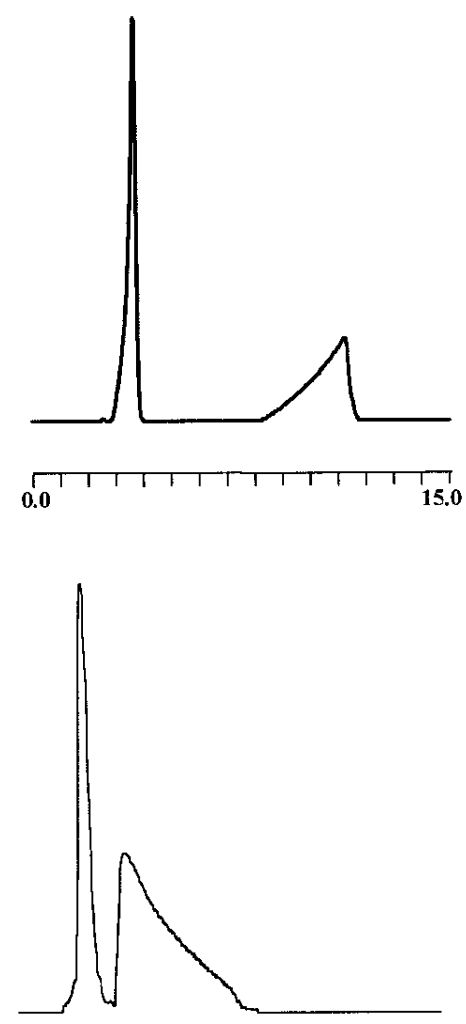

c.

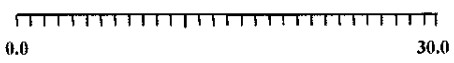

Figure 2. The effect of mobile phase additives on pindolol on Chiralcel $^{\circledR}$ OD. Mobile phase: a) methanol:diethylamine 99.9:0.1, $20{ }^{\circ} \mathrm{C}$; b) Hexane: Ethanol: Trifluoroacetic Acid 60:40:0.5, $40{ }^{\circ} \mathrm{C}$, load $0.01 \mathrm{mg}$. c) conditions as for b), but $25 \mathrm{mg}$ load. 
a.

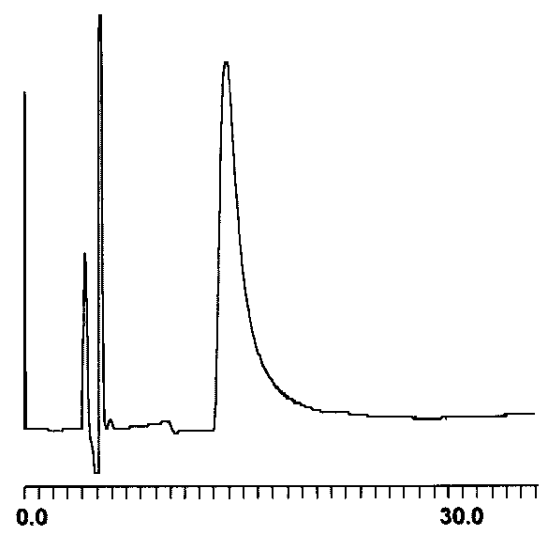

b.

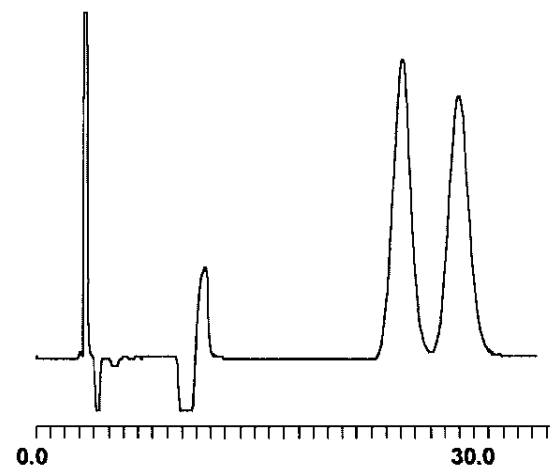

Figure 3. The effect of mobile phase additives on the chromatographic resolution of the enantiomers of albuterol. Mobile Phase: a) Hexane: ethanol: diethylamine, 93:7:0.1; b) hexane: ethanol: trifluoroacetic acid: triethylamine 95.5:4.5:0.1:0.17. Column Chiralcel ${ }^{\circledR}$ OJ.

on the optimisation of the separations to be carried out before this state of affairs can be reached. It is beyond the scope of this article to go deeply into chromatographic optimisation for large scale separations, but there are some influences of the CSP on scale up and these are, perhaps, relevant here.

\section{Influence of CSP cost on scale-up}

It is a sad fact of life that CSPs developed to date are relatively expensive. This may be due to several causes, but is related to the extra processing and costs beyond the simple preparation of a silica or a bonded phase silica packing material. As an example, the derivatised carbohydrate CSPs marketed by Daicel, which have been used to a large extent in both preparative and production scale enantioselective chromatography, are prepared starting from a bonded phase silica material. In addition, several other steps are required. A cellulose or amylose polymer of defined molecular weight distribution is first prepared. This is subsequently reacted exhaustively with an isocyanate (to prepare the carbamate derivative) or an acid chloride (for ester derivatives such as p-methylbenzoate). The resulting polymer is then coated in a multi-step process to achieve an even, controlled layer on the surface of the support. Extensive washing and conditioning steps are then carried out to ensure the complete removal of all reagents and to bring the polymer into the required tertiary structure which confers the maximum enantioselectivity. The end result of this processing is that the end cost of production of such materials is not very dependent upon the costs of production of the initial silica particles and is therefore largely independent of the diameter of the particles.

The particle size in preparative chromatography has been shown to be of little fundamental importance since as long as the required number of theoretical plates is achieved (by adjusting the column length) there is little to choose between the use of large and small particles, with the proviso that the larger particles can allow a slightly higher rate of production if operated at the same pressure as the smaller ones again with adjustment of the column length. When the costs of operation are considered, however, the effects of particle size can be more conclusive. For achiral separations, there is usually an optimum particle size. This is because the cost of the packing material is a strong function of the particle diameter. If a large particle size is used, the cost per kilo is small, but conversely, a long column is required which demands not only a large weight of packing (and thus a larger overall cost per column of the packing) but a larger hardware investment: a long high pressure column is more expensive than a short one. In enantioselective preparative chromatography, the CSP is almost without exception significantly more expensive than the corresponding achiral packing and, as noted, its cost does not change greatly with particle size. This means that to reduce the operating costs (and therefore the final production cost per $\mathrm{kg}$ of the desired enantiomer) it is essential to use the smallest quantity of CSP possible. This can only be achieved whilst maintaining the required number of theoretical plates for the separation by the use of small particle size packings. This is as true in SMB chromatography as for batch separations. Although SMB separations require fewer plates than are needed for the equivalent batch separation, the cost of the CSP imposes constraints on the equipment since the shortest possible columns need to be used in order to minimise it. There is always a compromise between operating pressure, solvent velocity, efficiency, particle size and column length. Currently both theoretical analysis and current manufacturing practice are converging upon particle diameters of the order of $20 \mu \mathrm{m}$ for the CSP required for SMB separations. If a smaller particle size is used the required column length becomes too small for the wide diameter columns required for production; a column $10 \times 80 \mathrm{~cm}$ is feasible, but to reduce this to $3 \mathrm{~cm}$ in length becomes impractical. If a larger particle size is used, the columns are forced to be made longer to achieve even the small numbers of plates required and thus the cost of CSP inventory increases, adding to the final cost of the product.

\section{Conclusion}

A variety of packing materials can be used very effectively for preparative and production scale chromatographic resolution of enantiomers. At the laboratory scale, it is important to have CSPs with as wide a range of applicability as possible in order both to minimise the investment in columns or packing materials and to be able quickly to find suitable 
conditions for the separations. At the larger scale, it should be possible to spend some time in optimising the CSP to some degree, although in practice such time is not always available. In this case, a suitable packing material should be chosen from those types known to give high loadability before spending time on optimisation of its structure.

\section{References}

1. Colin, H.; Hilaireau, P.; de Tournemire, J. $L C / G C$ 1990, 8(4), 301.

2. Guiochon, G.; Golshan Shirazi, S.; Katti, A. M. Fundamentals of Preparative and Nonlinear Chromatography, Academic Press, 1994; p 631.

3. Cox, G. B. in Process Scale Liquid Chromatography, Subramanian G. Ed., VCH, Weinheim, 1995.

4. Eble, J. E.; Grob, R. L.; Antle, P.E.; Snyder, L. R. J Chromatogr. 1987, 384, 25.

5. Jacobson, S.; Golshan-Shirazi, S.; Guiochon, G. A.I.Ch.E. J. 1991, 37, 836 .
6. Golshan-Shirazi, S.; Huang, J. -X.; Guiochon, G. Anal. Chem. 1991, 63, 1147.

7. Cox, G. B.; Snyder, L. R. J. Chromatogr. 1989, 483, 95.

8. Wülf, G.; Minarik, M. Chromatographic Chiral Separations, Zief M. and Crane L.J. Eds., Dekker, 1988.

9. Blaschke, G.; Schwanghart, A. -D. Chem. Ber. 1976, 109, 1967.

10. Pirkle, W. H.; House, D. W.; Finn, J. M. J. Chromatogr. 1980, 192, 143.

11. Francotte, E. J. Chromatogr. 1994, 666, 565.

12. Francotte, E. US Patent 5, 091, 520, 1992.

13. Ichida, A.; Shibata, T.; Okamoto, I.; Yuki, Y.; Namikoshi, H.; Toga, Y. Chromatographia 1984, 19, 280.

14. Geiser, F. unpublished results.

15. Geiser, F. HPLC 97, Birmingham.

16. Tang, Y. Chirality 1996, 8, 136-142.

17. Armstrong, D. W.; Zukowski, J. J. Chromatogr. 1994, 666, 445. 